\title{
Updating the grading criteria for adult diffuse gliomas: beyond the WHO2016CNS classification
}

\author{
Takashi Komori $^{1}$ \\ Published online: 14 February 2020 \\ (c) The Japan Society of Brain Tumor Pathology 2020
}

\section{Introduction}

The classification of adult diffuse gliomas has shifted from the traditional histogenetic classification to a molecular one in the updated 4th edition of the WHO Classification of Tumours of the Central Nervous System, published in 2016 (WHO2016CNS) [10]. This shift is based on the discovery of isocitrate dehydrogenase (IDH) mutations as the most upstream genetic event in two canonical diffuse glioma lineages: diffuse astrocytomas and oligodendrogliomas of adults (the "molecular era") [7, 8]. While this revision represents a paradigm shift in brain tumor classification, the grading schemes for diffuse gliomas were not modified simultaneously, as no alternative method that satisfactorily replaces the traditional histological grading approach using morphologic features (i.e. anaplasia, mitosis, microvascular proliferation [MVP] and necrosis has yet been reported).

To resolve such issues and recommend changes to the next WHO Classification update, the Consortium to Inform Molecular and Practical Approaches to CNS Tumor Taxonomy (cIMPACT-NOW) was established under the auspices of the International Society of Neuropathology [11]. Since then, five updates have been published, including grading criteria for adult diffuse astrocytic gliomas with IDH-wildtype and IDH-mutant; cIMPACT-NOW update 3 highlighted the molecular criteria that could be used for IDH-wildtype grade II/III astrocytomas harboring molecular feature of glioblastoma, IDH-wildtype [3], while update five proposed novel classifications and terminology for IDHmutant astrocytomas [4].

This editorial summarizes the remaining issues concerning the grading of IDH-mutant adult diffuse gliomas.

Takashi Komori

komori-tk@igakuken.or.jp

1 Department of Laboratory Medicine and Pathology (Neuropathology), Tokyo Metropolitan Neurological Hospital, 2-6-1 Musashidai, Fuchu, Tokyo 183-0042, Japan

\section{Definition of the WHO grade}

The WHO grade, which is printed in Roman numerals, has been defined as not pure histological grading but instead a malignancy scale of the expected clinical outcomes of patients across all tumor types based on the known natural history after surgical resection alone for each tumor entity. In addition, with the WHO CNS classification, a tumor with a different WHO grade is designated a different name, even within the same tumor type. For example, infiltrating low-grade astrocytoma, grade II, is named "diffuse astrocytoma", and infiltrating high-grade astrocytoma, grade III, is named "anaplastic astrocytoma", even though they are essentially an identical tumor type. One exception to this rule in WHO2016CNS is solitary fibrous tumor/hemangiopericytoma, for which three different grades can be assigned within the same tumor designation (grade 1, 2 and 3, printed in Arabic numerals) [10]. This principle of grading within a tumor entity is common in non-nervous system tumors.

However, it is extremely difficult to obtain an accurate natural history of IDH-mutant diffuse gliomas after surgical resection alone, as no historical data exist before the molecular era, and prospective data of those tumors, particularly higher-grade ones, are practically impossible to obtain, as these lesions are typically treated by either radiation and/or chemotherapy after resection. Due to these limitations, the grades of not only IDH-mutant gliomas but any tumor must be assigned based on retrospective studies.

\section{Issues in mitotic counting and proliferation indices}

In the current grading scheme, distinguishing between grade II and III diffuse gliomas depends on the presence of a significant number of mitoses as well as anaplasia. However, the WHO has never specified the actual cut-off number for differentiating grade II and III due to the non-reproducibility 
and inter-observer variability of the mitotic count, which may be caused by the following: discriminating mitoses, particularly granular ones, from apoptosis is often challenging; the specimen size varies; the diameter of the microscopic fields differs depending on the manufacturer; and the evaluation area may be different, whether evaluating 10 consecutive high-powered fields or an entire specimen. In practice, $>2$ mitoses as a cut-off value has been used, since the WHO 3rd edition (2000) adopted this criterion for anaplastic astrocytoma based on previous studies showing that a single mitosis does not allow for the diagnosis of anaplastic astrocytoma [6].

To overcome the above-mentioned issues associated with counting mitoses, immunostaining with $\mathrm{pHH} 3$ antibody, which can discriminate mitoses from apoptosis, is often applied. This immunostaining approach is so sensitive for identifying mitoses that it can convert a grade 2 lesion on conventional H\&E staining to a grade 3 lesion by identifying more mitoses in the same specimen. This antibody may also yield diffuse false positive staining, particularly when used with automated immune-stainers.

Since the discovery of IDH mutations, it has been suggested that the legacy grading scheme depending on the mitotic count may not be a strong predictor of the outcome of WHO grade II and III astrocytomas with IDH-mutant, although it is still considered valid for IDH-wildtype gliomas [12].

Similarly, studies of the proliferative index (e.g. based on Ki-67) have not adequately identified the threshold for stratifying the risk among patients with diffuse gliomas. One technical issue involves tissue section aging, which is a common problem associated with nuclear antigens (e.g. Ki-67, estrogen receptor, p53, etc.), as they are often weakened by a long storage duration and higher storage temperature [15]. Standardization as well as optimization of the antigen retrieval protocol for Ki-67 across every institution is generally much more challenging than standardizing the mitotic counting approach.

\section{CDKN2A/B homozygous deletion and other genetic alterations}

Multiple studies have shown that some tumors clearly demonstrate discrepancies among existing WHO grades; for instance, IDH-mutant astrocytomas may have a better prognosis than IDH-wildtype astrocytomas [5]. Interestingly enough, Shirahata et al. found that the overall survival of IDH-mutant diffuse astrocytic tumors was associated with the $C D K N 2 A / B$ homozygous deletion status and the presence of necrosis; patients with $C D K N 2 A / B$ homozygous deletion without necrosis, graded as WHO grade III anaplastic astrocytoma according to the current criteria, exhibited an equivalent survival rate to those with WHO grade IV glioblastoma, IDH-mutant with $C D K N 2 A / B$ homozygous deletion and necrosis [13]. In addition, the glioma cases with necrosis and no $C D K N 2 A / B$ homozygous deletion survived significantly longer than those with $C D K N 2 A / B$ homozygous deletion but no necrosis. They also found that the deletion did not exist in grade II astrocytoma. Although another study by Appay et al. confirmed this finding [2], Yang et al. identified this homozygous deletion in $12 \%$ of grade II tumors [16]. One possible reason for such a discrepancy is that while Shirahata et al. [13] used pHH3 antibody, which is sensitive as mentioned above, Yang et al. [16] did not; without using pHH3 antibody, they might have identified fewer mitoses, and thus regarded grade III tumors in other studies as grade II ones, resulting in a lower rate of grade III.

Regarding other alterations of RB pathway genes as well as other genetic alterations, including PIK3CA or PIK3RI mutations, PDGFRA amplification and MYCN amplification, the Consortium has failed to identify clinically relevant evidence to include in the updated grading approach because of the shortage of published data [3]. Similarly, genomic instability and reduced global DNA methylation may be markers for stratifying the risks of patients with gliomas, but methods of detecting these conditions are not firmly established to be the standard of grading scheme. The above histologic and genetic information are summarized in Figure 1.

\section{cIMPACT-NOW proposal for IDH-mutant astrocytomas}

Taken together, the cIMPACT-NOW update five proposes a novel grading approach incorporating $C D K N 2 A / B$ homozygous deletion into the WHO2016CNS criteria as follows [4]:

Astrocytoma, IDH-mutant, WHO grade 2: lacking significant mitoses, MVP, necrosis and $C D K N 2 A / B$ homozygous deletion

Astrocytoma, IDH-mutant, WHO grade 3: exhibiting anaplasia and significant mitoses but lacking MVP, necrosis and $C D K N 2 A / B$ homozygous deletion

Astrocytoma, IDH-mutant, WHO grade 4: exhibiting MVP or necrosis or $C D K N 2 A / B$ homozygous deletion or any combination of these features

With this new approach, the Consortium has abandoned the legacy principle of grading and terminology; first, grading within a tumor entity was adopted, i.e. "Astrocytoma, IDHmutant, WHO grade 4" is not as malignant as "Glioblastoma, IDH-wild-type, WHO grade 4"; second, three different grades are assigned within the same tumor designation, 


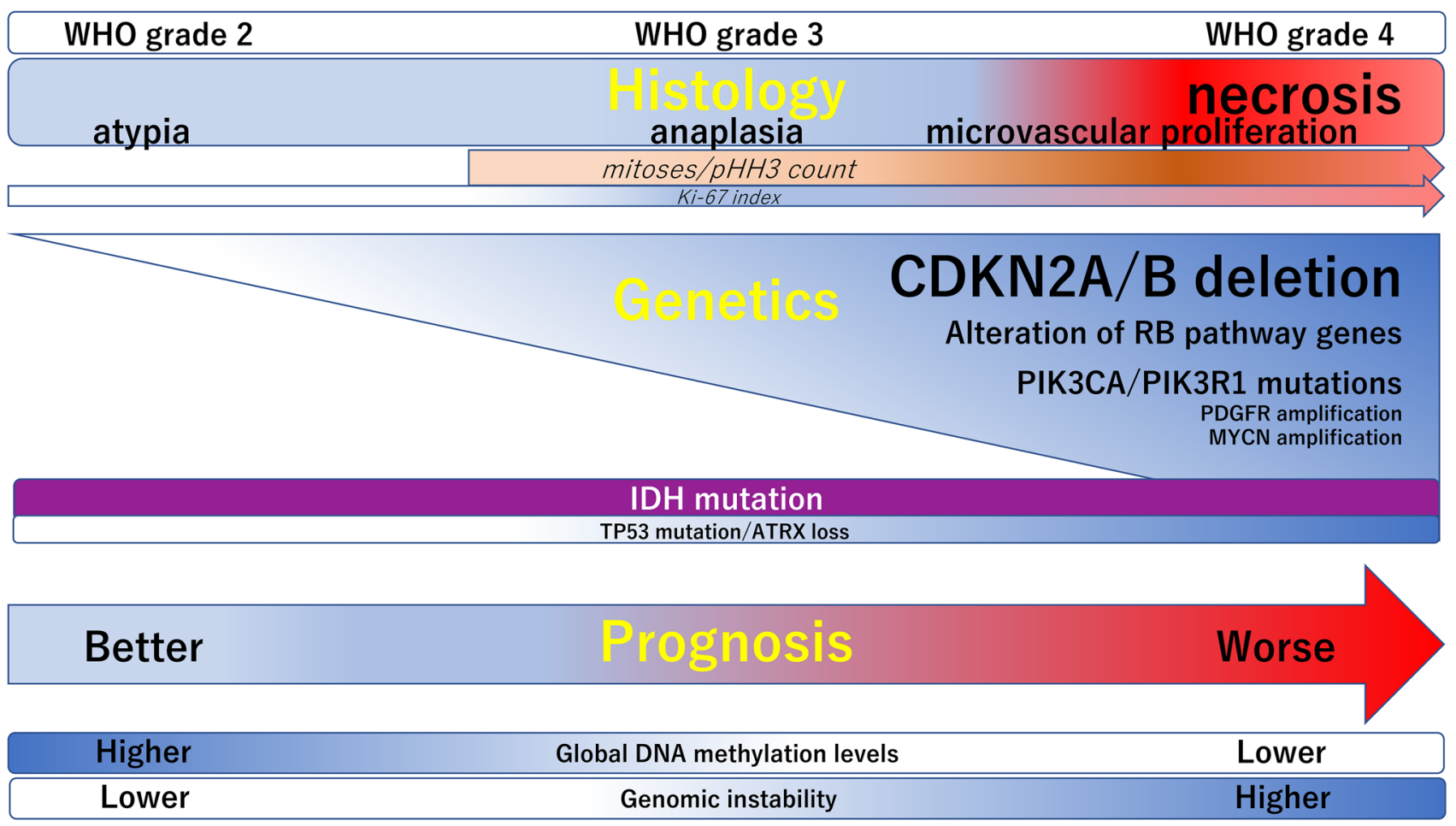

Fig. 1 Grading of IDH-mutant astrocytomas. While necrosis is still a significant histologic marker for a poor prognosis, the mitotic count and microvascular proliferation seem to be weaker predictors of the outcome. Multiple studies have shown $C D K N 2 A / B$ homozygous to
"Astrocytoma, IDH-mutant"; third, Arabic numerals are used instead of Roman numerals for the grade. Arabic numerals are the standard style in the upcoming WHO 5th edition [4].

\section{Grading issues in oligodendrogliomas, IDH-mutant and 1p/19q codeleted (codeleted oligodendrogliomas)}

While one study suggested that $C D K N 2 A$ homozygous deletion is a strong adverse prognostic factor in anaplastic codeleted oligodendroglioma [2], another showed that NOTCH1 mutations were associated with a shorter survival in codeleted oligodendroglioma [1], while no such association was found for $C D K N 2 A$ alterations. No other studies have confirmed those results or identified biomarkers that can convincingly distinguish lower- and higher-grade oligodendrogliomas [9]. The current grading approach thus remained unchanged.

\section{Summary}

At present, no single biomarker capable of adequately stratifying the risks of patients with diffuse adult gliomas exists. While the legacy grading approach remains useful for be a strong adverse prognostic marker in patients with IDH-mutant astrocytoma. Other markers listed have also been suggested as useful adverse prognostic markers, but further study is needed.

assessing the risk of IDH-wildtype astrocytomas, it seems less useful in cases of IDH-mutant gliomas; only necrosis is significant when integrated with $C D K N 2 A / B$ homozygous deletion. More studies need to be performed to address the above-mentioned grading issues, and alternative better scheme to define WHO grade would be required. For the time being, the integration of histology with clinical, radiological and designated molecular information remains the most promising way of making a proper diagnosis and ensuring the best patient care [14].

\section{References}

1. Aoki K, Nakamura H, Suzuki H, Matsuo K, Kataoka K, Shimamura T, Motomura K, Ohka F, Shiina S, Yamamoto T et al (2018) Prognostic relevance of genetic alterations in diffuse lower-grade gliomas. Neuro Oncol 20:66-77. https://doi.org/10.1093/neuonc/ nox 132

2. Appay R, Dehais C, Maurage CA, Alentorn A, Carpentier C, Colin C, Ducray F, Escande F, Idbaih A, Kamoun A et al (2019) CDKN2A homozygous deletion is a strong adverse prognosis factor in diffuse malignant IDH-mutant gliomas. Neuro Oncol 21:1519-1528. https://doi.org/10.1093/neuonc/noz124

3. Brat DJ, Aldape K, Colman H, Holland EC, Louis DN, Jenkins RB, Kleinschmidt-DeMasters BK, Perry A, Reifenberger G, Stupp R et al (2018) cIMPACT-NOW update 3: recommended 
diagnostic criteria for "diffuse astrocytic glioma, IDH-wildtype, with molecular features of glioblastoma, WHO grade IV". Acta Neuropathol 136:805-810. https://doi.org/10.1007/s0040 1-018-1913-0

4. Brat DJ, Aldape K, Colman H, Figrarella-Branger D, Fuller GN, Giannini C, Holland EC, Jenkins RB, Kleinschmidt-DeMasters B, Komori T et al (2020) cIMPACT-NOW update 5: recommended grading criteria and terminologies for IDH-mutant astrocytomas. Acta Neuropathol. https://doi.org/10.1007/s00401-020-02127-9

5. Cancer Genome Atlas Research N, Brat DJ, Verhaak RG, Aldape KD, Yung WK, Salama SR, Cooper LA, Rheinbay E, Miller CR, Vitucci M et al (2015) Comprehensive, integrative genomic analysis of diffuse lower-grade gliomas. N Engl J Med 372:2481-2498. https://doi.org/10.1056/NEJMoa1402121

6. Giannini C, Scheithauer BW, Burger PC, Christensen MR, Wollan PC, Sebo TJ (1999) Cellular proliferation in pilocytic and diffuse astrocytomas. J Neuropathol Exp Neurol. https://doi. org/10.1097/00005072-199901000-00006

7. Iuchi T, Sugiyama T, Ohira M, Kageyama H, Yokoi S, Sakaida T, Hasegawa Y, Setoguchi T, Itami M (2018) Clinical significance of the 2016 WHO classification in Japanese patients with gliomas. Brain Tumor Pathol 35:71-80. https://doi.org/10.1007/s1001 4-018-0309-0

8. Komori T (2017) The 2016 WHO Classification of Tumours of the Central Nervous System: the major points of revision. Neurol Med Chir (Tokyo) 57:301-311. https://doi.org/10.2176/nmc. ra.2017-0010

9. Komori $\mathrm{T}$ (2019) The anticipated revision of the grading criteria for adult isocitrate dehydrogenase-mutant diffuse glioma within the neuro-oncology community. Neuro Oncol 21:1485-1486. https://doi.org/10.1093/neuonc/noz186

10. Louis DN, Perry A, Reifenberger G, von Deimling A, FigarellaBranger D, Cavenee WK, Ohgaki H, Wiestler OD, Kleihues P, Ellison DW (2016) The 2016 World Health Organization classification of tumors of the central nervous system: a summary. Acta Neuropathol 131:803-820. https://doi.org/10.1007/s0040 1-016-1545-1
11. Louis DN, Aldape K, Brat DJ, Capper D, Ellison DW, Hawkins C (2017) Announcing cIMPACT-NOW: the consortium to inform molecular and practical approaches to CNS tumor taxonomy. Acta Neuropathol. https://doi.org/10.1007/s00401-016-1646-x

12. Olar A, Wani KM, Alfaro-Munoz KD, Heathcock LE, van Thuijl HF, Gilbert MR, Armstrong TS, Sulman EP, Cahill DP, VeraBolanos E et al (2015) IDH mutation status and role of WHO grade and mitotic index in overall survival in grade II-III diffuse gliomas. Acta Neuropathol 129:585-596. https://doi.org/10.1007/ s00401-015-1398-z

13. Shirahata M, Ono T, Stichel D, Schrimpf D, Reuss DE, Sahm F, Koelsche C, Wefers A, Reinhardt A, Huang K et al (2018) Novel, improved grading system(s) for IDH-mutant astrocytic gliomas. Acta Neuropathol 136:153-166. https://doi.org/10.1007/s0040 1-018-1849-4

14. Sonoda Y, Yokoo H, Tanaka S, Kinoshita M, Nakada M, Nishihara $\mathrm{H}$, Committee for molecular diagnosis of the Japan Society of Brain Tumor P (2019) Practical procedures for the integrated diagnosis of astrocytic and oligodendroglial tumors. Brain Tumor Pathol 36:56-62. https://doi.org/10.1007/s10014-019-00337-y

15. Wester K, Wahlund E, Sundstrom C, Ranefall P, Bengtsson E, Russell PJ, Ow KT, Malmstrom PU, Busch C (2000) Paraffin section storage and immunohistochemistry. Effects of time, temperature, fixation, and retrieval protocol with emphasis on p53 protein and MIB1 antigen. Appl Immunohistochem Mol Morphol 8:61-70

16. Yang RR, Shi ZF, Zhang ZY, Chan AK, Aibaidula A, Wang WW, Kwan JSH, Poon WS, Chen H, Li WC et al (2019) IDH mutant lower grade (WHO Grades II/III) astrocytomas can be stratified for risk by CDKN2A, CDK4 and PDGFRA copy number alterations. Brain Pathol. https://doi.org/10.1111/bpa.12801

Publisher's Note Springer Nature remains neutral with regard to jurisdictional claims in published maps and institutional affiliations. 